\title{
Activated gp130 signaling selectively targets B cell differentiation to induce mature lymphoma and plasmacytoma
}

\author{
Anna K. Scherger, ${ }^{1}$ Mona Al-Maarri, ${ }^{2}$ Hans C. Maurer, ${ }^{3}$ Markus Schick, ${ }^{1}$ Sabine Maurer, ${ }^{1}$ \\ Rupert Öllinger, ${ }^{3,4,5}$ Irene Gonzalez-Menendez, ${ }^{6}$ Manuela Martella, ${ }^{6}$ Markus Thaler, ${ }^{7}$ \\ Konstanze Pechloff, ${ }^{4,7,8}$ Katja Steiger, ${ }^{8,9}$ Sandrine Sander, ${ }^{10}$ Jürgen Ruland, ${ }^{4,7,8}$ Roland Rad, ${ }^{3,4,5,8}$ \\ Leticia Quintanilla-Martinez, ${ }^{6}$ Frank T. Wunderlich, ${ }^{2}$ Stefan Rose-John, ${ }^{11}$ and Ulrich Keller ${ }^{1,8,12}$ \\ ${ }^{1}$ Internal Medicine III, Technische Universität München, Munich, Germany. ${ }^{2}$ Max Planck Institute for Metabolism \\ Research, Center for Endocrinology, Preventive Medicine and Diabetes, Cologne, Germany. ${ }^{3}$ Internal Medicine II, ${ }^{4}$ Center \\ for Translational Cancer Research, and ${ }^{5}$ Institute of Molecular Oncology and Functional Genomics, Technische Universität \\ München, Munich, Germany. ${ }^{6}$ Institute of Pathology, Eberhard-Karls-Universität Tübingen, Tübingen, Germany. ${ }^{7}$ Institute \\ of Clinical Chemistry and Pathobiochemistry, Technische Universität München, Munich, Germany. ${ }^{8}$ German Cancer \\ Consortium and Cerman Cancer Research Center, Heidelberg, Germany. ${ }^{9}$ Institute of Pathology, Technische Universität \\ München, Munich, Germany. ${ }^{10}$ Adaptive Immunity and Lymphoma, German Cancer Research Center/National Center for \\ Tumor Diseases Heidelberg, Heidelberg, Germany. "Institute of Biochemistry, Christian-Albrechts-Universität zu Kiel, \\ Kiel, Germany. ${ }^{12}$ Department of Hematology, Oncology and Tumor Immunology (Campus Benjamin Franklin), Charité - \\ Universitätsmedizin Berlin, Germany.
}

\begin{abstract}
Aberrant activity of the glycoprotein 130 130/JAK/STAT3 (gp130/JAK/STAT3) signaling axis is a recurrent event in inflammation and cancer. In particular, it is associated with a wide range of hematological malignancies, including multiple myeloma and leukemia. Novel targeted therapies have only been successful for some subtypes of these malignancies, underlining the need for developing robust mouse models to better dissect the role of this pathway in specific tumorigenic processes. Here, we investigated the role of selective gp130/JAK/STAT3 activation by generating a conditional mouse model. This model targeted constitutively active, cell-autonomous gp130 activity to $B$ cells, as well as to the entire hematopoietic system. We found that regardless of the timing of activation in B cells, constitutively active gp130 signaling resulted in the formation specifically of mature B cell lymphomas and plasma cell disorders with full penetrance, only with different latencies, where infiltrating $\mathrm{CD} 138^{+}$cells were a dominant feature in every tumor. Furthermore, constitutively active gp130 signaling in all adult hematopoietic cells also led to the development specifically of largely mature, aggressive B cell cancers, again with a high penetrance of CD138+ tumors. Importantly, gp130 activity abrogated the differentiation block induced by a B cell-targeted Myc transgene and resulted in a complete penetrance of the gp130-associated, CD138+, mature B cell lymphoma phenotype. Thus, gp130 signaling selectively provides a strong growth and differentiation advantage for mature $B$ cells and directs lymphomagenesis specifically toward terminally differentiated $B$ cell cancers.
\end{abstract}

Authorship note: AKS and MAM contributed equally to this study.

Conflict of interest: The authors have declared that no conflict of interest exists.

Copyright: (c) 2019, American Society for Clinical Investigation.

Submitted: February 25, 2019

Accepted: July 9, 2019

Published: August 8, 2019.

Reference information: JCl Insight. 2019;4(15):e128435. https://doi. org/10.1172/jci.insight.128435.

\section{Introduction}

Interleukin 6 (IL-6) is a pleiotropic cytokine various cell types secrete during infection, inflammation, and cancer. IL-6 binds to the IL-6 receptor (IL-6R) to form an inactive IL-6/IL-6R complex that associates with the membrane glycoprotein 130 (gp130), which is ubiquitously expressed and functions as the signal-transducing receptor partner of the IL-6R (1). Downstream signaling induced by IL-6/ IL-6R/gp130 is crucial for regulating B cell and T cell responses and for coordinated activity of both the innate and adaptive immune systems. Accordingly, IL-6/IL-6R/gp130 activity fulfills both homeostatic functions as well as crucial proinflammatory actions during pathogen infection (2). Also, gp130 complexes with other IL-6-type cytokine receptors, thereby initiating several key intracellular signaling pathways, including the PI3K/AKT, MAPK, and JAK/STAT3 pathways, to regulate many cellu- 
lar processes. For example, the consequence of STAT3 activation is the context-dependent expression of a cast of target genes that mediate physiological functions, including regulation of cell proliferation and differentiation, cell migration, cell survival, and angiogenesis (3-7). As a consequence of these diverse functions, aberrant signaling downstream of gp130 has been associated with a wide variety of diseases, including rheumatoid arthritis (8) and a range of hematological malignancies (reviewed in ref. 9). Therefore, a detailed mechanistic understanding of gp130's diverse functions in specific cell types could have direct clinical implications.

IL-6 was initially described as "B cell differentiation factor" based on its importance for B cell differentiation as well as proliferation and immunoglobulin (Ig) production by plasma cells $(10,11)$. Multiple gp130 signaling cytokines induce proliferation of B cells (12-14), and consistently, mice with mutations in gp130-mediated signaling and IL-6-deficient mice are impaired in developing germinal centers (GCs) and generate defective antibody responses (15-17). These effects are mediated by STAT3, which is activated by IL- 6 and IL-21 to enhance expression of B cell lymphoma 6 and induce the generation of T follicular helper cells, both mandatory for a functional GC reaction. STAT3 activation also regulates the expression of the transcription factor B lymphocyte-induced maturation protein 1 (Blimp1), which promotes terminal differentiation of B cells into long-lived plasma cells that produce high amounts of Ig (18). Consistently, C57BL/6 mice overexpressing IL-6 develop plasmacytosis (19); however, plasma cell tumors (plasmacytomas) are seen only on a BALB/c genetic background (20). This strain-specific phenomenon is mediated by mutations in the INK4a/ARF locus, which supports lymphoma- and myelomagenesis (21). Thus, alternative in vivo models are needed for characterizing the mechanisms of lymphoma and myeloma pathogenesis seen in patients and for providing novel tools for testing therapies.

We previously developed an in vivo tool to study the role of membrane-independent activation of STAT3, which is seen in many neoplastic diseases. This tool, leucine zipper plus gp130 (L-gp130), has the entire extracellular portion of gp130 replaced with the dimerizing human c-Jun leucine zipper sequence, thereby forcing gp130 dimerization, resulting in cell-autonomous and constitutive activation of associated intracellular signaling independent of cytokine stimulation (22). A bone marrow (BM) transduction-transplantation model of L-gp130 in vivo developed a malignancy mimicking human multiple myeloma (MM) (23), a plasma cell neoplasm that originates in the BM (24). However, whole-BM infection can also activate the proinflammatory pathway downstream of active STAT3, which was linked to tumorigenesis. The importance of IL-6/IL-6R/gp130 downstream signaling in lymphoid cell malignancies, such as large cell lymphoma and Hodgkin lymphoma, is also supported by oncogenic aberrations resulting in STAT3 activation, as well as activating mutations in JAKs and STAT3 (25-27).

Advanced mature B cell malignancies, including MM, represent a wide range of frequent cancers and are still considered incurable for many patients. Despite significant progress in understanding the pathogenesis of these malignancies, novel targeted therapies have been successful for only some subentities and specific scenarios (28). To develop alternative preclinical models, we here generated a mouse model on a C57BL/ 6 background where conditional L-gp130 activation was directed to the hematopoietic system at different developmental stages. Activation of aberrant gp130/JAK/STAT3 signaling at distinct stages of B cell development generated mice with a range of highly aggressive, mature B cell lymphomas and plasmacytoma with full penetrance. These mice presented signaling pathway signatures closely mimicking advanced human B cell malignancies that could be used as preclinical models to better evaluate candidate therapies.

\section{Results}

An in vivo model for conditional gp130 activation. We generated a mouse model to study the in vivo effects of aberrant activity of IL-6/IL-6R/gp130/JAK/STAT3 signaling. We designed a transgene that allows conditional expression of L-gp130 by generating a ROSA26 knockin mouse strain where compound L-gp130 and Zoanthus sp. green fluorescent protein (ZsGreen) expression from the chicken- $\beta$-actin promoter with CMV-immediate early enhancer (CAG) promoter is prevented by a locus of recombination by Cre-flanked (loxP-flanked) and a locus of recombination by Dre-flanked (rox-flanked) stop cassette (Figure 1A). Crossing these mice to deleter Dre mice removed the rox-flanked stop cassette in the germline, thereby generating a Cre-dependent L-gp130 allele. Clone C6 was selected for generation of the L-gp130 mouse strain (Figure 1B).

To test whether Cre-mediated L-gp130 activation resulted in activation of gp130 downstream signaling, murine embryonic fibroblasts (MEFs) from E13.5 L-gp $130^{f /+}$ embryos were infected with a retrovi- 
rus encoding Cre recombinase (Cre-IRES-YFP). C57BL/6 WT MEFs infected with a retrovirus encoding either L-gp130 (L-gp130-IRES-GFP, positive control) or GFP control (MSCV-IRES-GFP, MIG, negative control) as well as $L$-gp $130^{t /+}$ MEFs infected with MIG were used as controls. L-gp130 expression activated STAT3 (measured as phospho-STAT3) as compared with the negative control (Figure 1, C and D).

Constant gp130 signaling promotes B cell differentiation and the GC reaction. To investigate whether forced activation of gp130 downstream signaling pathways promotes B cell differentiation and proliferation, $L$-gp$130^{f /+}$ mice were bred to $C D 19 \mathrm{Cre}^{+/-}$mice, targeting L-gp130 expression specifically to B lymphocytes (29). $C D 19 C r e^{+/-} L-g p 130^{t /+}(C D 19 L-g p)$ and $L-g p 130^{t /+}(L-g p)$ control mice were sacrificed at the age of 6 weeks and further analyzed. Immunoblot analysis of purified B cells revealed L-gp130 expression and subsequent aberrant STAT3 activation in B cells derived from CD19 L-gp mice (Supplemental Figure 1A; supplemental material available online with this article; https://doi.org/10.1172/jci.insight.128435DS1). Gross physical examination did not show any obvious pathology, and spleens did not differ in weight between groups (Supplemental Figure 1B). Moreover, histological examination of spleens and BM displayed largely unaltered morphology and cellularity (Supplemental Figure 1C), and absolute white blood cell (WBC) numbers were comparable (Supplemental Figure 1D). Analysis of the T cell $\left(\mathrm{CD}^{+}\right)$and B cell $\left(\mathrm{B} 220^{+}\right)$compartments in BM, spleen, and peripheral blood (PB) of young CD19 L-gp and age-matched L-gp control mice by flow cytometry revealed no significant changes in T and B cell frequencies (Supplemental Figure 1E).

We next investigated the consequences of constitutive gp130 activation in B cells in more detail. Supplemental Figure 2 shows the flow cytometry gating strategy used throughout this study. Flow cytometry analysis displayed a significant increase of mature $\mathrm{IgD}^{+} \mathrm{B}$ cells in the $\mathrm{PB}$, spleen, and $\mathrm{BM}$ in young $C D 19$ L-gp mice compared with controls, in which $\operatorname{IgM}^{+} \mathrm{B}$ cells representing an immature stage of B cell differentiation were increased (Figure 2A).

Mining publicly available data for STAT3 targets during B cell development, we identified the highest expression in plasmablasts and plasma cells in accordance with high STAT3 activity, while STAT3 expression itself diminished during late B cell differentiation. IL-6/JAK/STAT3 signaling activity was highest in plasma cells and memory B cells (Figure 2B). To investigate differences in B cells derived from either young CD19 L-gp or control mice, we performed RNA sequencing (RNA-Seq) followed by a principal component analysis (PCA). This analysis revealed that L-gp130-expressing B cells clustered differently from control B cells (Figure 2, C and D, and Supplemental Figure 1F). Specifically, B cells from young CD19 L-gp mice displayed a significant activation of IL-6/JAK/STAT3, PI3K/AKT/mTOR, WNT, Myc, as well as B cell receptor (BCR) and NF- $\mathrm{kB}$ signaling, which we defined as the "L-gp130 signature" (Figure 2E and Supplemental Methods). To determine whether this signature was also present in humans, we investigated distinct B cell stages during late differentiation. We found this L-gp130 signature most prominent in human GC B cells (Supplemental Figure 1G).

Altogether, these results link constitutively activated gp130 signaling in B cells with several pathways associated with STAT3 and B cell proliferation, B cell differentiation, and BCR signaling activity.

Activation of gp130 signaling in $B$ cells results in mature $B$ cell malignancies. Having identified that activation of gp130/JAK/STAT3 signaling via B cell-specific L-gp130 expression increased the percentage of mature B cells in young CD19 L-gp mice, we monitored an aging cohort. CD19 L-gp mice showed deterioration of health at a median of 151 days and were sacrificed in accordance with animal investigation regulations. $C D 19 \mathrm{Cre}^{+/-}(C D 19)$ as well as L-gp control mice did not develop disease signs within the observed time frame (Figure 3A). The visible disease pattern comprised mainly tumors originating from the mesenteric lymph nodes (LNs), splenomegaly, and occasionally axillary as well as inguinally enlarged LNs. To investigate the exact cause of illness, CD19 L-gp mice underwent whole-body necropsy and extended flow cytometric analysis of hematopoietic organs (Supplemental Figure 2). B cell-specific activation of gp130 downstream signaling resulted in B cell malignancies with a penetrance of $100 \%$. When analyzing the mesenteric nodal tumors, the compartment of highest tumor manifestation, 2 subtypes of ZsGreen ${ }^{+}$ $\mathrm{B}$ cell disorders were identified in this cohort as depicted in Figure 3B: the first was identified as a plasma cell (PC) disorder characterized by the presence of $\mathrm{CD} 138^{+} \mathrm{CD} 19^{-}$cells. The second represented CD19+ $\mathrm{B}$ cell tumors characterized by strong IgD expression and was therefore termed mature B cell lymphoma (Mature). The 2 remaining mice were defined as having B cell lymphoma (Other). Fractions of the 3 B cell cancers identified are shown in Figure 3C. Flow cytometric results were confirmed by histological and immunohistochemical analyses, where the tumors arising could also be divided into 2 major groups. Importantly, CD $138^{+}$PCs were present in tumors of all subgroups (Figure 3D), indicating a possible con- 
A Rosa26 WT locus

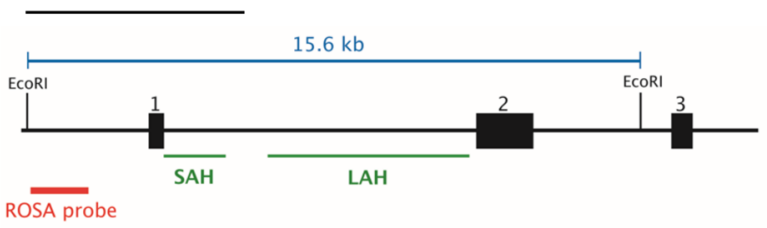

B9/36 L-gp130 targeting vector

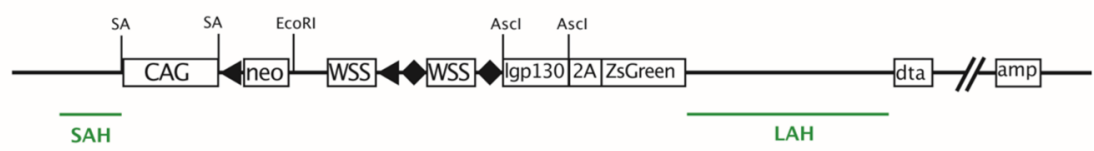

after homologous recombination

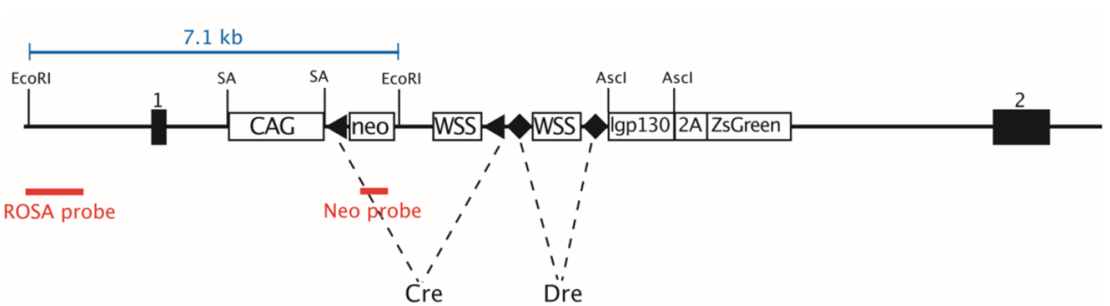

B

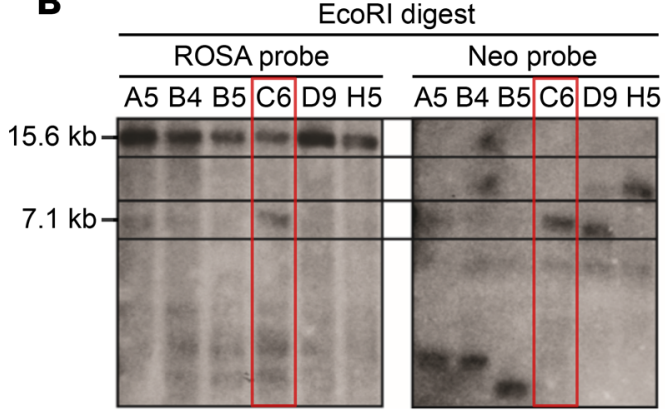

C

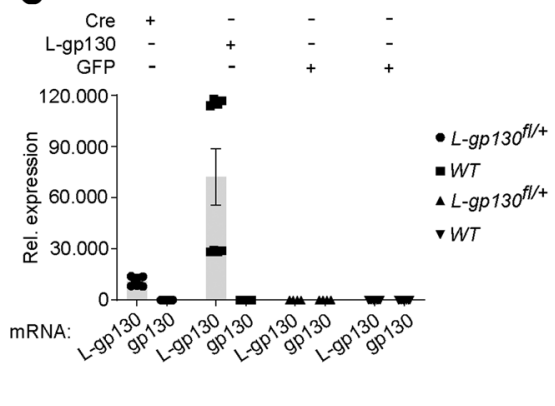

D

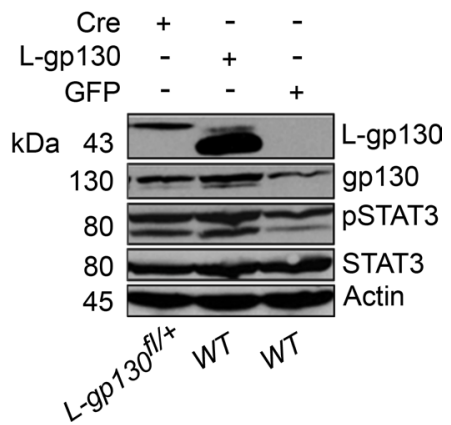

Figure 1. In vivo model for conditional gp130 activation. (A) Strategy to insert the floxed L-gp130-2A-ZsGreen cassette under the control of the CAC promoter into the mouse ROSA26 locus. The targeted locus is depicted before and after homologous recombination. EcoRI sites within the targeted genomic region are indicated. Amp, ampicillin resistance gene; CAG, chicken- $\beta$-actin promoter with CMV-immediate early enhancer; DTA, diphtheria toxin fragment A; LAH, long arm of homology; L-gp130, leucine zipper plus glycoprotein 130; loxP, locus of recombination by Cre; neo, neomycin resistance gene; rox, locus of recombination by Dre; SAH, short arm of homology; SV-40, simian virus 40; WSS, Westphal stop sequence; ZsGreen, Zoanthus sp. green fluorescent protein. (B) Southern blot analysis of targeted embryonic stem cell clones. The EcoRI-Pacl external probe was used to screen EcoRI-digested clonal DNA via Southern blot. Besides the 15.6-kb WT band, a 7.1-kb targeted band appeared in correctly targeted clones. To verify single integration, a radioactively labelled probe was removed from the Southern blot membrane, which was reprobed with internal neo probe showing a single 7.1-kb band in single integrated clones. Clone C6 was selected for generation of the L-gp130 mouse strain. (C) MEFs of indicated genotypes (symbols) were infected with the designated virus(es) and sorted for GFP/YFP positivity. Depicted is the mRNA expression for L-gp130 and gp130 relative to GAPDH. Shown are means \pm SEM. (D) Immunoblot analysis of MEFs from C using the indicated antibodies. L-gp130 was detected using a gp130 antibody.

tinuum within the L-gp130-induced disease with malignant transformation occurring at various stages of B cell development. The transcription factor Pax5 is expressed in B cells from the pro-B cell to the mature B cell stage but is absent in terminally differentiated PCs (30). Immunohistochemistry revealed Pax5 staining in the Mature phenotype whereas tumors of the PC phenotype lacked Pax5 (Figure 3D). In line with results from flow cytometry (Figure 3B), staining for IgM was barely present in the Mature and PC phenotypes (Figure 3D). Other organs, i.e., spleen and BM, of diseased mice were also infiltrated by transformed cells (Supplemental Figure 3A). However, we saw a difference in cells infiltrating the different organs when comparing diseased mice of the PC and the Mature phenotypes. Besides infiltrating mes- 


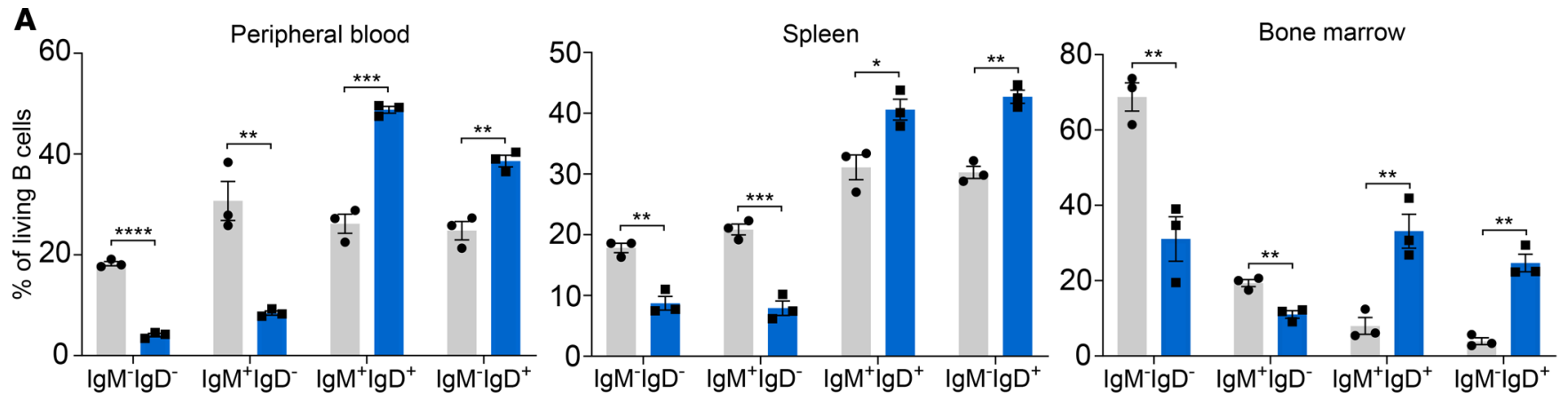
L-gp
CD19;L-gp ZsGreen ${ }^{+}$

B

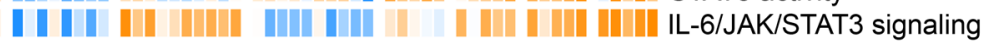
|||||| ||| |||||| |||||| ||||| || STAT3 expression

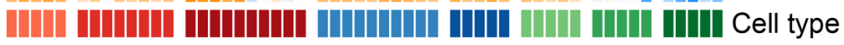
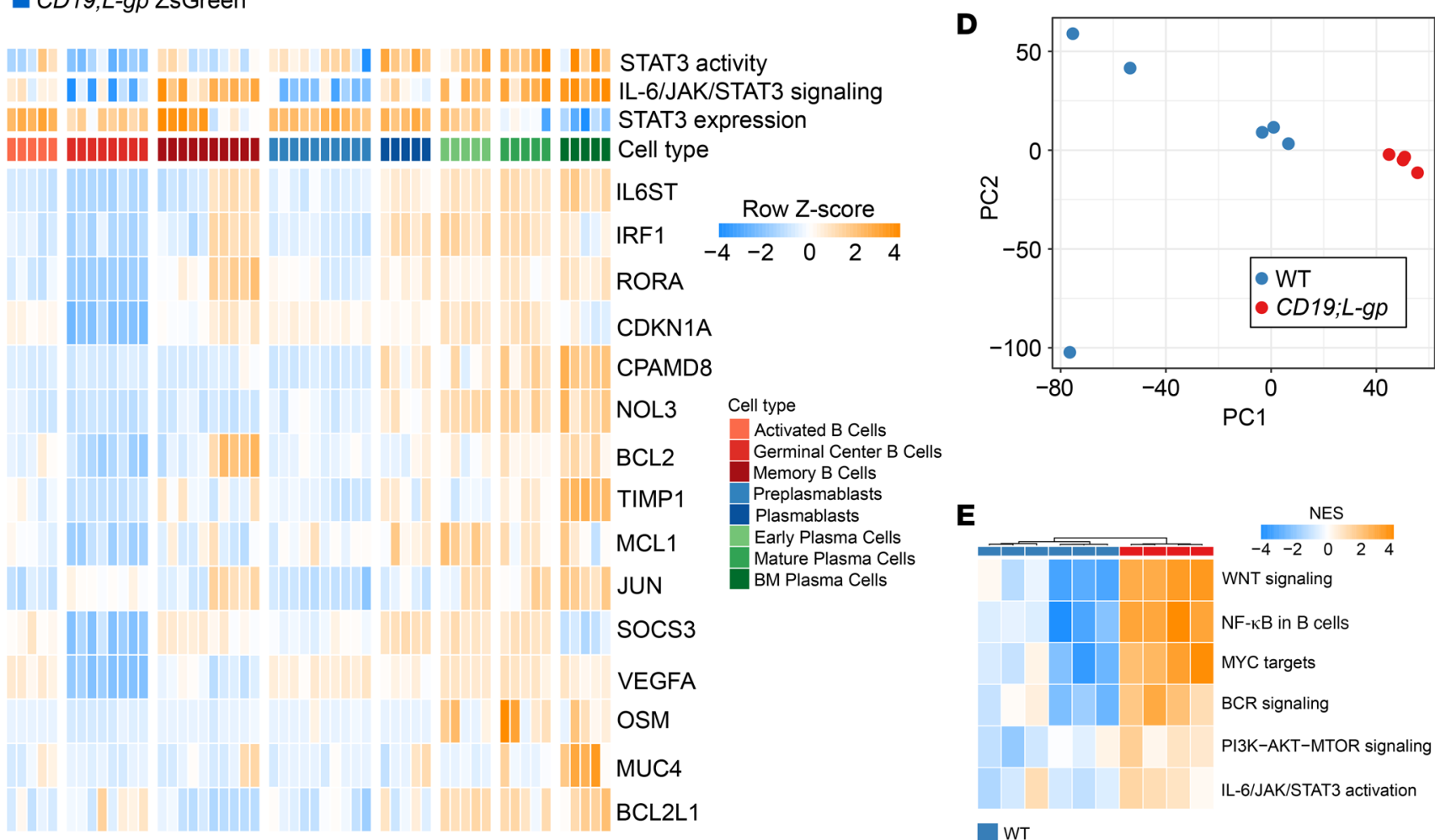

PI3K-AKT-MTOR signaling IL-6/JAK/STAT3 activation

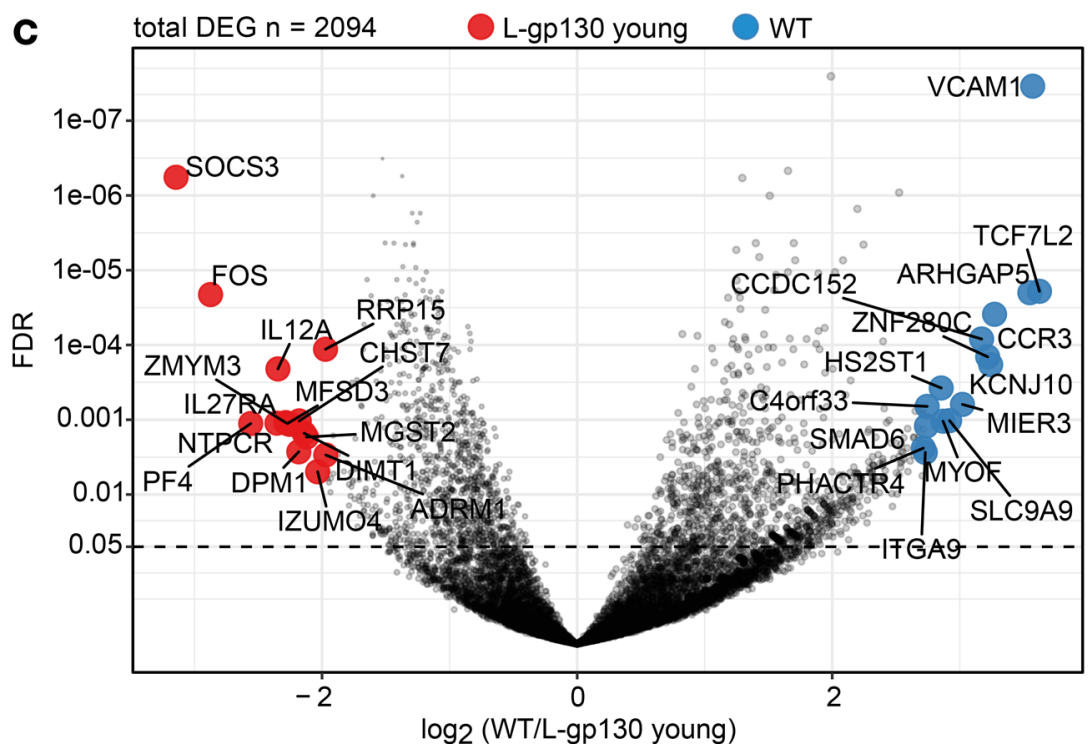


Figure 2. Activated gp130 signaling promotes B cell differentiation and signaling associated with oncogenic pathways. (A) Flow cytometry analysis of $\mathrm{PB}$, spleen, and BM reveal a significant accumulation of mature IgD+ B cells within the ZsGreen+ population of CD19 L-gp mice while L-gp controls present with an immature lgM ${ }^{+}$phenotype $(n=3) .{ }^{*} P<0.05,{ }^{* *} P<0.005,{ }^{* *} P<0.0005$, and ${ }^{* * *} P<0.0001$ as determined by 2 -tailed Student's $t$ test. Shown are means \pm SEM. (B) Heatmap depicting the expression of STAT3 target genes among various stages of B cell development. The upper part of the panel illustrates the respective cell type as well as results for mere STAT3 expression (third row from top) as opposed to STAT3 activity and IL-6/JAK/STAT3 pathway activity (top and second row from top, respectively) as determined by single-sample gene set enrichment analysis. (C) Volcano plot illustrating results from a differential gene expression analysis between CD19+ splenocytes isolated from WT and young CD19 L-gp mice. The top 15 up- (blue) and downregulated (red) genes are labeled. Positive $\log _{2}$ fold changes indicate higher levels in WT splenocytes. A total of 2094 differentially expressed genes (DEGs) were identified at an FDR $\leq 0.05$. (D) PCA of RNA-Seq gene expression profiles obtained by isolating CD19+ splenocytes from WT (blue) and young CD19 L-gp (red) mice, respectively. (E) Heatmap depicting single-sample gene set enrichment results as normalized enrichment scores (NESs) for the indicated pathways and their distribution between CD19+ splenocytes isolated from WT (blue) and young CD19 L-gp (red) mice. All pathways shown are differentially enriched at an FDR $<0.1$.

enteric nodal tumors, $\mathrm{CD} 138^{+} \mathrm{CD} 19^{-}$also often manifested within the $\mathrm{BM}$ while the spleens were hardly infiltrated by these cells of the PC phenotype (Supplemental Figure 3B, left). In the Mature phenotype, however, $\mathrm{CD} 19^{+} \mathrm{IgD}^{+} \mathrm{B}$ cells were seen equally in $\mathrm{BM}$ and spleens in addition to infiltrating mesenteric nodal tumors (Supplemental Figure 3B, right). A CD138 $\mathrm{CD} 19^{+}$plasmablastic cell population hardly occurred in sick CD19 L-gp mice, with only 5 out of the 48 analyzed animals (i.e., 10.4\%) displaying infiltration of this population. However, $\mathrm{CD} 138^{+} \mathrm{CD} 19^{+}$plasmablastic cells were seen only in the spleen and sometimes $\mathrm{BM}$ of these diseased mice at low percentages and were missing in the nodal tumors (Supplemental Figure 3C). In addition to phenotypic analysis by flow cytometry, we further assessed the clonality status in the diseased animals. Whereas only $31.3 \%$ of mice that were assigned to the PC group showed serum electrophoresis indicative of monoclonal gammopathy, more than half (55.3\%) of all mice with a Mature phenotype presented with this characteristic (Figure 3E). In accordance with clonality analysis of mesenteric nodal tumors by VDJ-PCR revealing that the majority were monoclonal (Figure 3F), analysis of Ig serum levels showed frequently elevated IgG levels in mice of both phenotypes within the CD19 L-gp cohort (Supplemental Figure 3D). This was also observed when performing RNA-Seq analysis for expression of Ig heavy constant gamma chains that encode for the IgG isotypes (Supplemental Figure 3E). In addition, pronounced differences were also seen for expression of the remaining heavy chains defining the IgA, IgD, and IgM isotypes (Supplemental Figure 3E). Light chain constant region gene expression revealed a shift toward higher $\kappa$ and lower $\lambda$ gene expression in the $C D 19$ L-gp mice of both phenotypes as compared with WT controls (Supplemental Figure 3F). Serial transplantation of tumor cells from sick mice with a PC phenotype into sublethally irradiated syngeneic recipients resulted in disease reestablishment with reproduction of the $\mathrm{CD} 138^{+}$phenotype (Supplemental Figure 3, G and $\mathrm{H}$ ).

Thus, activation of constitutive gp130 signaling very early during B cell development resulted in mature B cell neoplasms and PC disorders with $100 \%$ penetrance.

gp130 activation during or after GC B cell differentiation results in delayed transformation. Activating L-gp130 in B cells using CD19Cre led to mature B cell lymphoma and PC cancers. To identify the precise role of activated gp130 signaling in B cell transformation, we next took advantage of additional mouse strains that allow conditional gene activation within the GC and toward the PC stage, namely $C \gamma 1 C r e$ mice (31) and PRDM1Cre (referred to as Blimp1Cre) (32), respectively. As previously seen in CD19 L-gp mice, both C $\gamma 1 C r e^{+/-}$

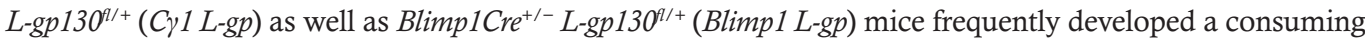
disease and presented with mesenteric nodal tumors. The estimated median survival for $C \gamma 1 \mathrm{~L}$-g $p$ mice was 629 days (Figure 4A), whereas Blimp1 L-gp mice died significantly earlier (median survival 329 days, Figure 4B). In addition to the PC and Mature phenotype in diseased CD19 L-gp mice, an additional phenotype was found in $C \gamma 1 \mathrm{~L}-g p$ with accumulation of $\mathrm{CD} 19^{+} \mathrm{GL} 7^{+} \mathrm{GC}$ B cells, thus termed GC phenotype (Figure 4C). Figure 4D shows the distribution of the 3 phenotypes within the Cy 1 L-gp cohort. Diseased Blimp 1 L-gp mice presented with PC and Mature phenotypes, affirming that $\mathrm{B}$ cell transformation occurred subsequent to the GC reaction upon activation of gp130/JAK/STAT3 (Figure 4, E and F). Immunohistochemical analysis of tumors from diseased C $1 \mathrm{~L}$-gp and Blimp1 L-gp mice of the PC phenotype revealed high infiltration of $\mathrm{CD} 138^{+}$cells (Figure 4G). CD138 ${ }^{+}$cells were also seen in GC tumors from $C \gamma 1 \mathrm{~L}-\mathrm{gp}$ mice when stained for immunohistochemical analysis. However, the infiltration rate of these cells in the GC phenotype of $C \gamma 1 \mathrm{~L}-g p$ mice was considerably lower as compared with the PC phenotype (Figure 4G). Other organs, i.e., spleen and $\mathrm{BM}$, of diseased mice were also infiltrated by tumor cells (Supplemental Figure 4A), most of them to a lesser extent (Supplemental Figure 4B). Several mice of both genotypes showed serum gammopathy indicating Ig 
A
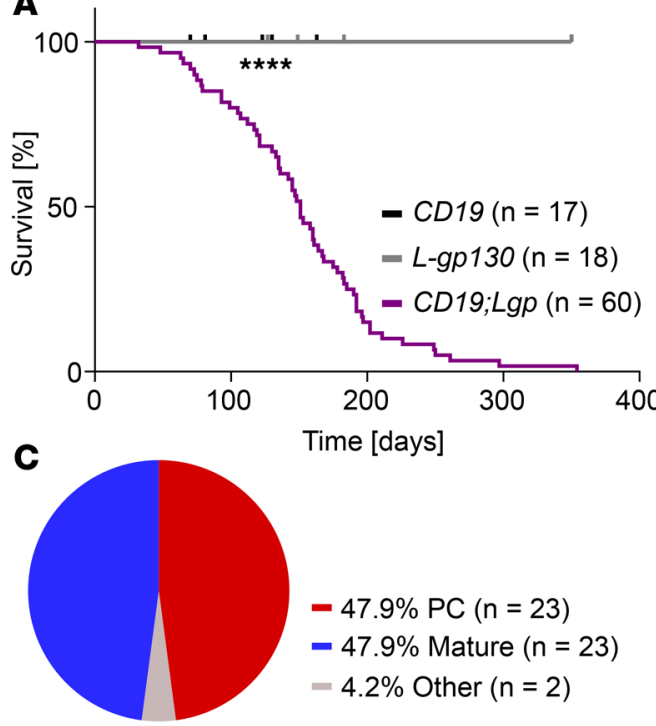

Time [days]

$-47.9 \%$ PC $(n=23)$

$-47.9 \%$ Mature $(n=23)$

$-4.2 \%$ Other $(n=2)$
B

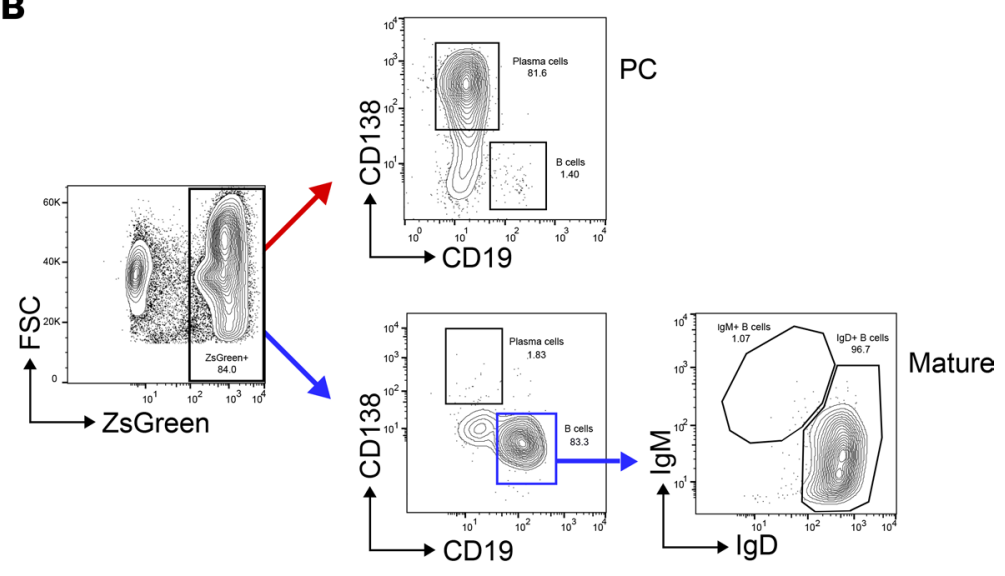

D

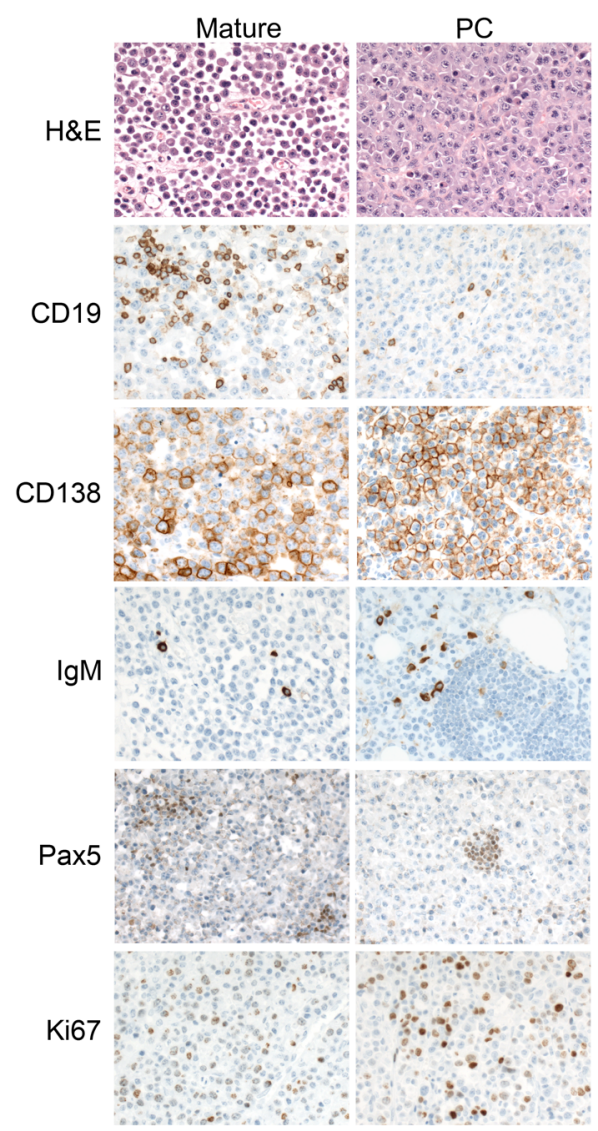

E
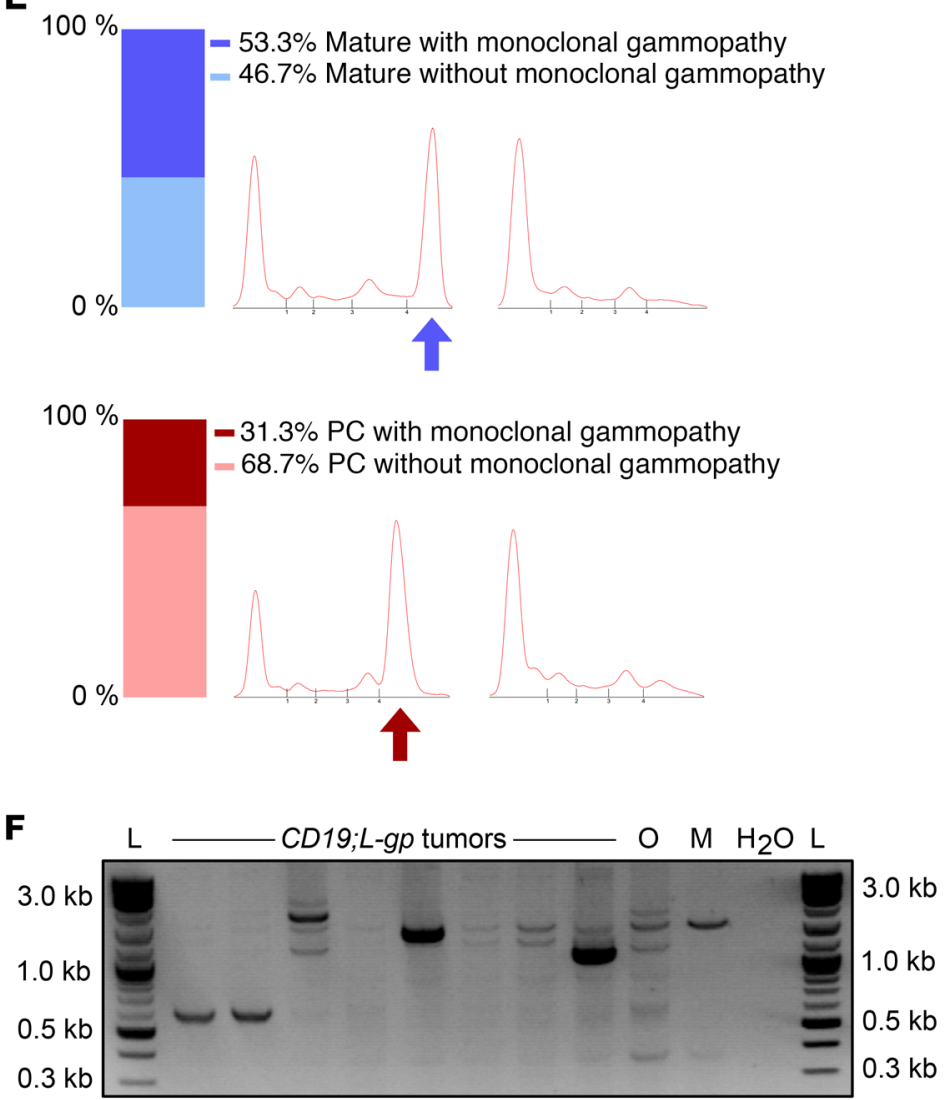

Figure 3. Activation of gp130 signaling targeted to B cells results in B cell malignancies. (A) Kaplan-Meier curve showing survival of CD19 L-gp mice $(n=60)$ in comparison with CD19 $(n=17)$ and L-gp $(n=18)$ controls. CD19 L-gp mice showed a median survival of 151 days, while controls did not show any signs of malignancy during the observed time frame. (*** $P<0.0001$, Mantel-Cox test). (B) Flow cytometric gating strategy applied on mesenteric nodal tumors for the 2 major phenotypes arising in CD19 L-gp mice. After exclusion of propidium iodide-positive ( $\left.\mathrm{PI}^{+}\right)$dead cells, the gate was set for ZsGreen+ cells to include all cells with activated JAK/STAT3 signaling. The first group was defined by CD19-CD138 ${ }^{+}$staining as a plasma cell (PC) phenotype and the second as a CD19+lgD+ mature B cell (Mature) phenotype. (C) Distribution of the spectrum of $B$ cell malignancies in diseased CD19 L-gp mice as determined by flow cytometry. The majority of diseased mice accounted for the PC or Mature phenotype, while 2 animals died of another B cell condition defined as Other; 48 mice were analyzed. (D) Histological and immunohistochemical analysis of 1 representative mesenteric nodal tumor of each indicated phenotype arising in CD19 L-gp mice. Note that CD138+ cells are present in both phenotypes. Original magnification, $\times \mathbf{4 0 0}$. At least 10 mice per phenotype were analyzed. (E) Serum protein electrophoresis from representative diseased $C D 19$ 
L-gp mice indicative of monoclonal gammopathy. A total of 31 mice were analyzed. Red and blue arrows point to the increased gamma-fraction, the characteristic of monoclonal gammopathy ("M-spike"). (F) Clonality analysis from CD138+ isolated tumor DNA of CD19 L-gp mice. Most tumors were monoclonal and only some represented an oligoclonal disease. O, oligoclonal control; $\mathrm{M}$, monoclonal control.

production (Supplemental Figure 4C). Accordingly, we detected significantly elevated IgG levels in $C \gamma 1 \mathrm{~L}-g p$ mice compared with $L$-gp controls while IgM levels were similar between groups (Supplemental Figure 4D). These data confirm the development of terminally differentiated B cell cancers by oncogenic gp130 activation at the GC or post-GC stage. Because constant L-gp130-induced signaling mainly activates IL-6/JAK/ STAT3, we interrogated publicly available gene expression data sets to analyze the activity of signaling in diverse disease stages of $\mathrm{MM}$ in comparison with monoclonal gammopathy of undetermined significance (MGUS) and normal PCs. We thereby found the IL-6/JAK/STAT3 signature most prominent in patients suffering from relapsed MM (Supplemental Figure 5).

In summary, gp130 downstream signaling at the GC and after GC B cell stages resulted in mature B cell cancers with an additional GC phenotype upon $C \gamma 1$ re introduction, indicating that IL-6/IL-6R/gp130 downstream signaling is crucial for mature B cell lymphomagenesis and that associated pathways are putative targets for therapeutic interventions.

Activated gp130 abolishes the lymphoma phenotype of B cell-targeted Myc and enforces plasmacytic differentiation. In healthy B cells, Myc expression triggers GC commitment: after Myc is suppressed in the GC dark zone, an increase in Myc expression in the light zone selects B cells for reentry into the GC dark zone and further affinity maturation (33). MYC aberrations frequently occur in GC and post-GC B cell lymphomas and are associated with adverse outcomes $(34,35)$. To determine whether this mechanism is influenced by gp130 signaling, we used $E \mu-M y c$ mice (36), where a $M Y C$ transgene is targeted to B cells, resulting in pre-B cell lymphoid tumors. We generated triple-transgenic mice of the CD19Cre L-gp130 E E-Myc (CD19 L-gp $M y c)$ genotype and control mice (CD19 L-gp and $M y c$ ). Only 1 triple-transgenic mouse was born alive out of 61 littermates ( $1.6 \%$ vs. $25 \%$ expected) but died at the age of 13 days. Consequently, we generated fetal liver hematopoietic stem/progenitor cell (FL-HSPC) grafts from E13.5 embryos at the expected Mendelian frequency. CD19 L-gp Myc and control FL-HSPC grafts were transplanted into lethally irradiated syngeneic recipient mice (Figure 5A). The median survival after transplantation was 20 days for CD19 L-gp Myc recipients, whereas recipients of control grafts survived significantly longer (CD19 L-gp, 265 days; Myc, 277 days) (Figure 5B). Triple-transgenic animals as well as $M y c$ controls presented with enlarged LNs and splenomegaly, while CD19 L-gp controls displayed only tumors of mesenteric LNs. Flow cytometry of diseased CD19 L-gp Myc animals revealed the same phenotype as seen in the single surviving triple-transgenic mouse, characterized by accumulating CD $19^{+} \mathrm{GL}^{+} \mathrm{GC}$ B cells and CD $138^{+} \mathrm{CD} 19^{-} \mathrm{PC}$ (Figure 5, C and D). The $M y c$ group succumbed to an immature B cell disease characterized by $\mathrm{CD}_{19}{ }^{+} \mathrm{IgM}^{+} \mathrm{IgD}^{-}$surface expression, while mature B cell subsets were completely absent (Figure 5, C and D). In contrast, the CD19 L-gp group developed a mature $\mathrm{B}$ cell disorder characterized by an accumulation of $\mathrm{IgD}^{+} \mathrm{B}$ cells (Figure $5 \mathrm{D}$ ). Flow cytometric results were confirmed by immunohistochemical staining of tumor material from $C D 19 L-g p$ $M y c$ as well as CD19 L-gp and Myc control mice. CD19 L-gp Myc-transplanted animals displayed intensive CD138 staining while lacking B220 expression whereas CD19 L-gp controls stained positive for both markers. In Myc controls, CD138 ${ }^{+}$staining was completely absent (Figure 5E). In contrast with Myc controls, CD19 L-gp Myc-as well as CD19 L-gp-transplanted mice showed only minor Myc and Pax5 staining (Figure $5 \mathrm{E})$. Although dying significantly later, $M y c$ controls demonstrated higher expression of the proliferation marker Ki67 in nodal tumor cells than CD19 L-gp Myc tumors (Figure 5E). Tumor infiltration was also identified in spleens and BM of diseased mice of all genotypes (Supplemental Figure 6).

Thus, activation of gp130 downstream signaling solely in B cells dominantly progresses the pro-/pre-B cell lymphoma phenotype characteristics of $E \mu-M y c$ mice toward a mature B cell/PC disorder. These data further support a key role of gp130 signaling in plasmacytoma and myeloma pathogenesis.

gp130 activation in HSPCs results in a highly aggressive B cell malignancy. Data presented heretofore indicated that activated constitutive gp130 signaling at distinct stages of B cell development drives differentiation of late B cells and ultimately leads to highly aggressive mature B cell lymphomas and PC tumors. However, JAK/STAT3 activation is a recurrent event in other hematopoietic neoplasms (reviewed in refs. 37, 38). Therefore, to ultimately test the significance of constitutive gp130 activation for promoting mature B cell lymphoma and plasmacytoma, we targeted genetic activation of the L-gp130 transgene to the entire hematopoietic system using $\operatorname{Vav} 1 \mathrm{Cre}(39,40)$. 
A

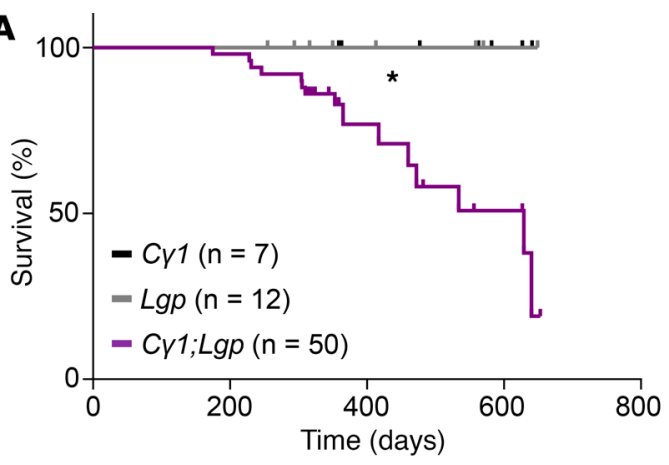

C
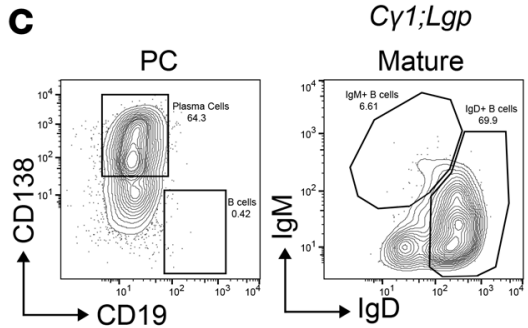

CY1;Lgp

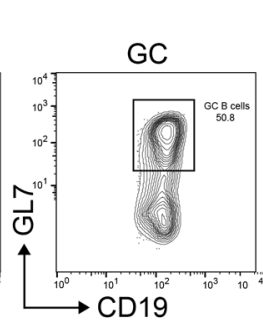

B

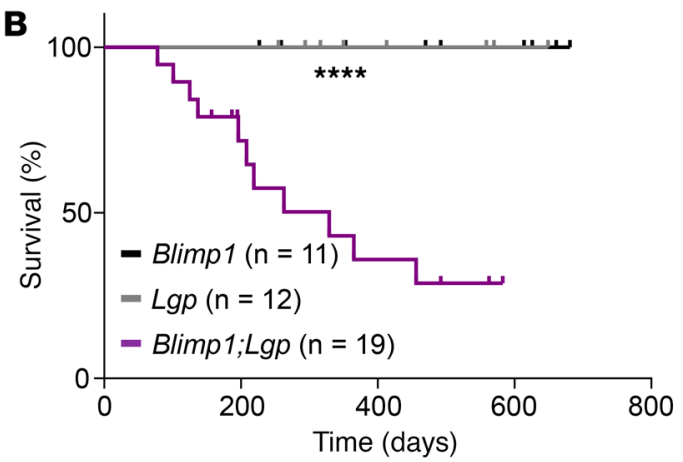

E

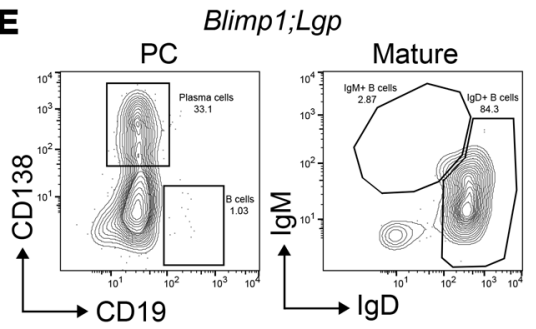

D

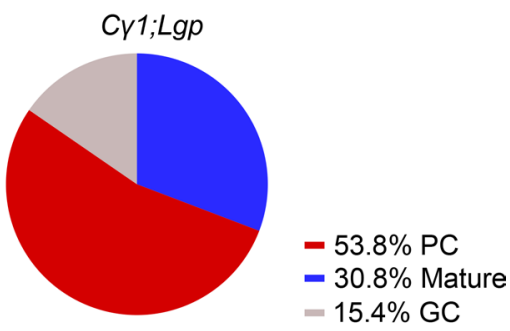

G

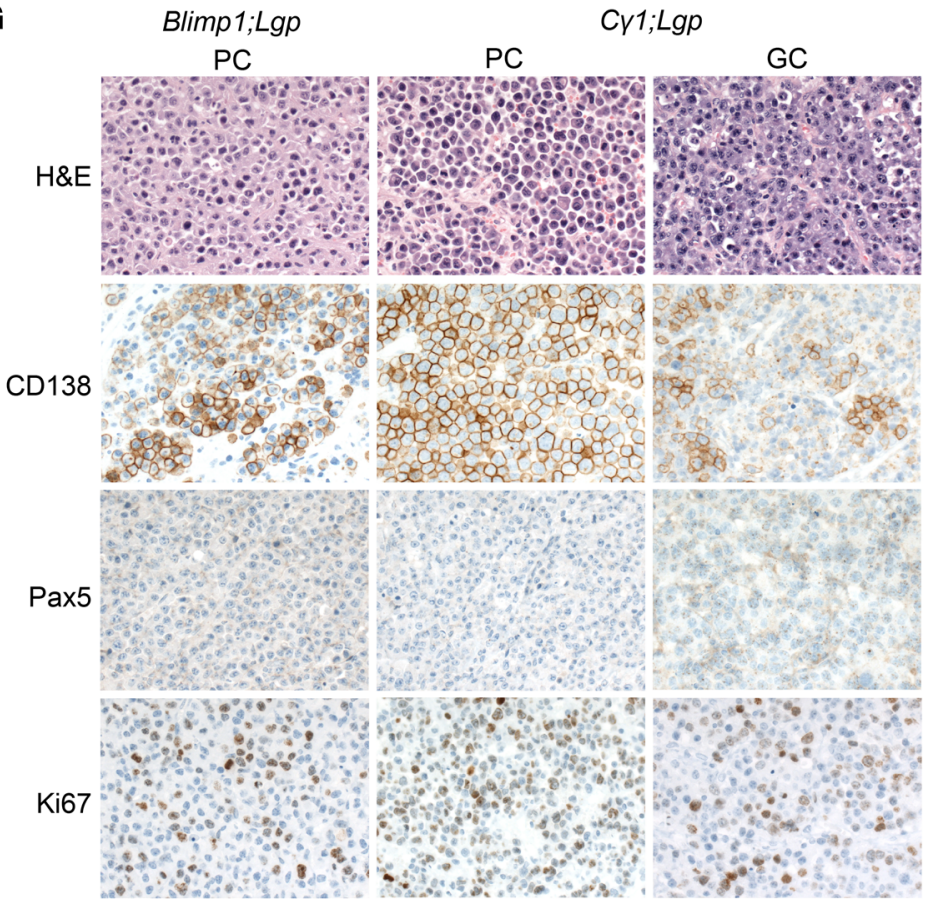

F Blimp1; Lgp

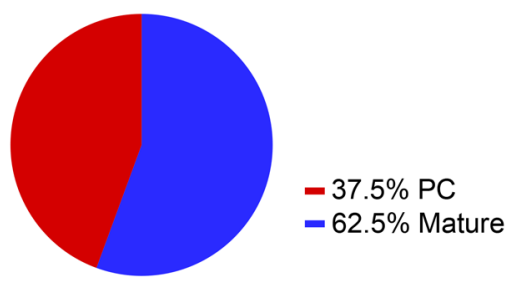

Figure 4. gp130 activation during or after CC B cell differentiation results in lymphoma and plasmacytoma. (A) Kaplan-Meier curve showing survival of C $\gamma 1 \mathrm{~L}$-gp mice $(n=50)$ in comparison with $C_{\gamma 1}(n=7)$ and L-gp $(n=12)$ controls. The median survival of $C \gamma 1$ L-gp compound mice was 629 days. Controls did not show any signs of malignancy during the observed time frame $\left({ }^{*} P=\right.$ 0.0109 , Mantel-Cox test). (B) Kaplan-Meier curve showing survival of Blimp1 L-gp mice $(n=19)$ in comparison with Blimp1 $(n=11)$ and $\operatorname{L-gp}(n=12)$ controls. The median survival of Blimp1 L-gp mice was 329 days. Controls did not show any signs of disease during the observed time frame $\left({ }^{* * *} P<0.0001\right.$, Mantel-Cox test). (C) Flow cytometric analysis from mesenteric nodal tumors of diseased C 11 L-gp mice identified 3 cohorts: CD19-CD138+ (PC, left), $\mathrm{CD} 19^{+} \mathrm{gDD}^{+}$mature $\mathrm{B}$ cell (Mature, middle), and $\mathrm{CD} 19^{+} \mathrm{CL} 7^{+}$ GC B cells (GC, right). Shown is the analysis of mesenteric nodal tumor material from 1 representative animal per phenotype. At least 3 mice per group were analyzed. (D) Distribution of the 3 types of B cell malignancies in diseased C $\gamma 1$ L-gp mice. (E) Flow cytometric analysis from diseased Blimp1 L-gp mice identified a CD19-CD138+ (PC) phenotype (left) and a CD19+lgD+ mature $B$ cell phenotype (Mature, right). Shown is the analysis of mesenteric nodal tumor material from 1 representative animal per phenotype. At least 3 mice per phenotype were analyzed. (F) Distribution of the 2 types of $B$ cell malignancies in diseased Blimp1 L-gp mice. (C) Histological and immunohistochemical analysis of 1 representative mesenteric nodal tumor from Blimp1 L-gp (left) and $C \gamma 1$ L-gp (middle and right) mice. Tumors with the $P C$ phenotype showed high infiltration of malignant cells positive for CD138 (left and middle). The GC phenotype displays only minor infiltration of CD138+ cells (right). At least 3 mice per genotype were analyzed. Original magnification, $\times 400$. 
A

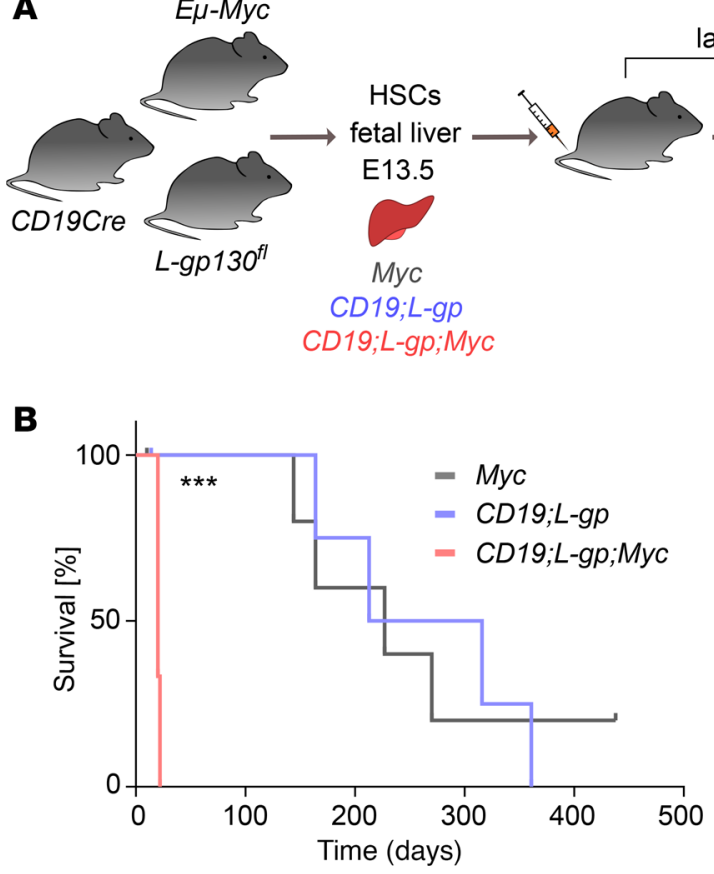

D
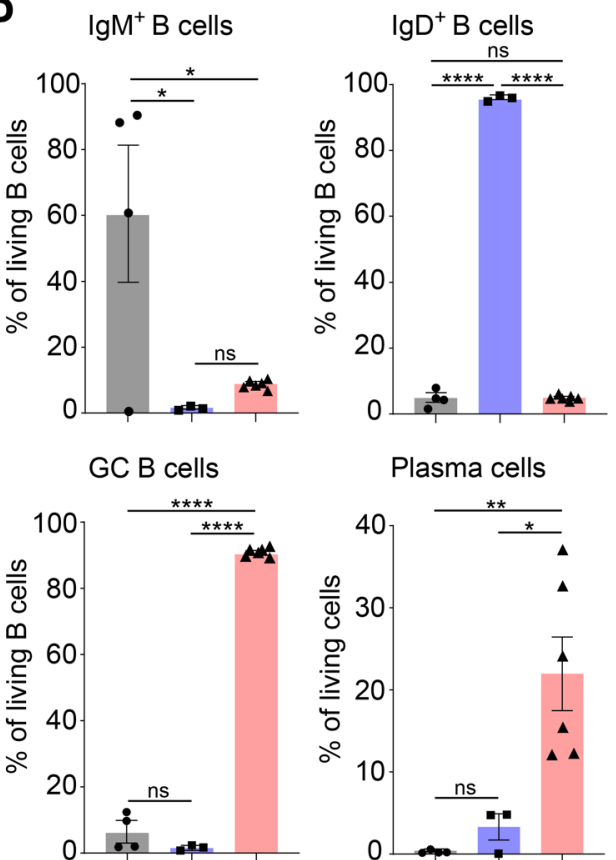

Plasma cells

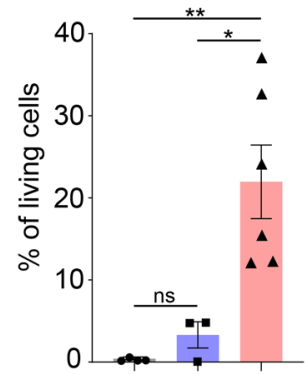

Myc

CD19;L-gp ZsGreen ${ }^{+}$

CD19;L-gp;Myc ZsGreen ${ }^{+}$

C
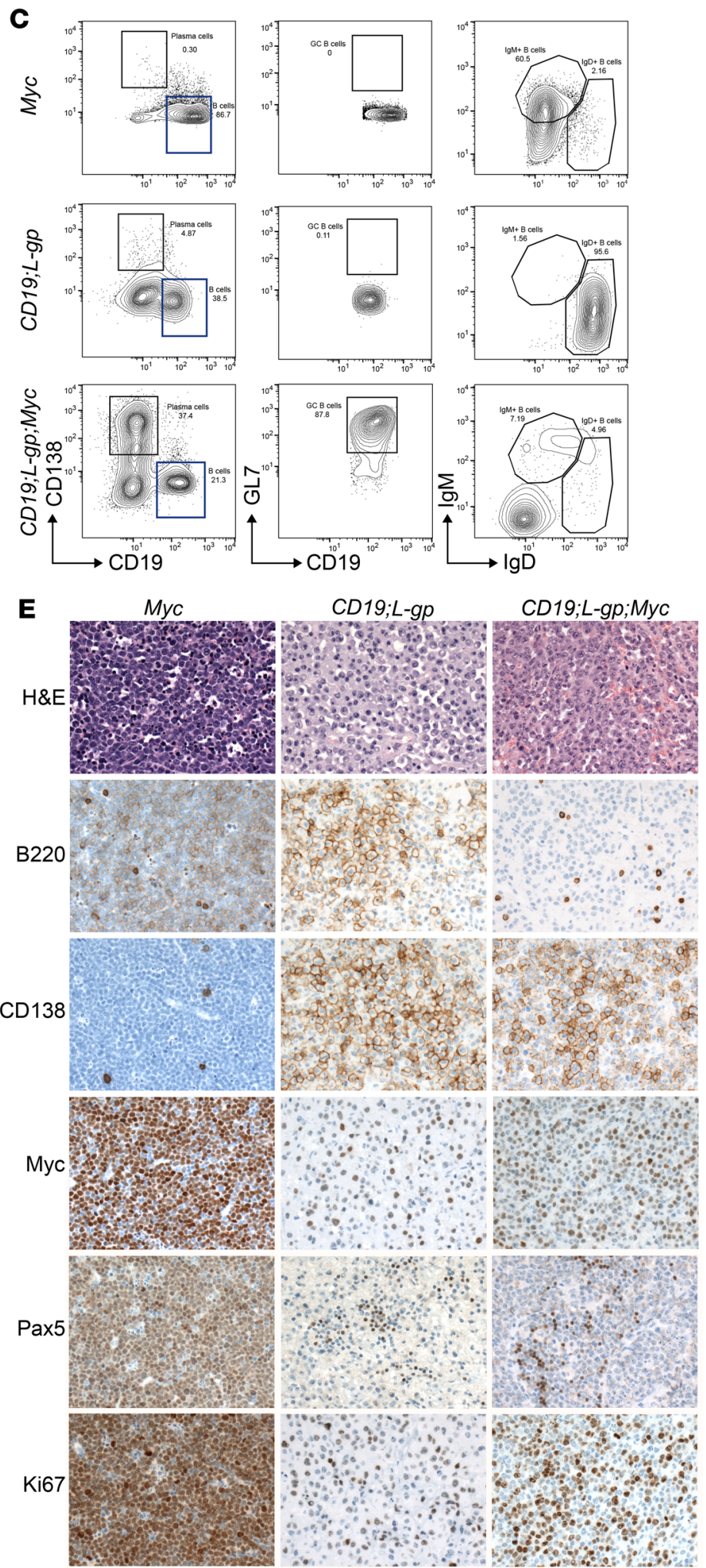
Figure 5. Activated gp130 abolishes the lymphoma phenotype of B cell-targeted Myc by enforcing plasmacytic differentiation. (A) Schematic of the CD19 L-gp Myc fetal liver cell (FLC) transplantation. FLCs from triple-transgenic CD19 L-gp Myc embryos as well as from CD19 L-gp and Myc controls were isolated at day 13.5 of embryonic development. Then, $1.2 \times 10^{6} \mathrm{FLCs}$ with $0.2 \times 10^{6}$ Ly5.1 BM support cells were transplanted into lethally irradiated C57BL/ 6 recipients. The mice were sacrificed upon disease onset and whole-body necropsy was performed. The data shown are from 1 representative experiment from a group size of $n=6$ animals each. (B) Kaplan-Meier curve revealing a significantly shorter survival of mice transplanted with FLCs from CD19 L-gp Myc as compared with controls ( $n=6$ mice per group) ( ${ }^{* *} P=0.003$, Mantel-Cox test). (C) Flow cytometric analysis of tumors. Tumors from CD19 L-gp Myc FLC-transplanted animals showed an accumulation of CD19-CD138+ PCs whereas this population was absent in CD19 L-gP and Myc control mice. At least 3 mice per genotype were analyzed. Shown are representative analyses. (D) Analysis of organ infiltration in CD19 L-gp Myc, CD19 L-gp, and Myc FLC-transplanted animals by distinct B cell populations as assessed by flow cytometry. Data are shown as mean percentage \pm SEM assessed from at least 3 mice per genotype. ${ }^{*} P<0.05,{ }^{* *} P<0.005,{ }^{* * *} P<0.0001$ by 2 -way ANOVA test. (E) Histology and immunohistochemistry from nodal tumor material of 1 representative animal per group. CD19 L-gp Myc-transplanted mice showed high positivity for CD138 staining while lacking B220 expression, whereas CD138+ cells are completely absent in the Myc control. The highest Myc, Pax5, and Ki67 expression was seen in Myc controls. Depicted in the figures are results obtained from analysis of the inguinal and mesenteric LNs, respectively; 3 mice per genotype were analyzed. Original magnification, $\times 400$.

Vav1Cre $e^{+/-} L-g p 130^{t /+}(\operatorname{Vav} 1 \mathrm{~L}-g p)$ mice rapidly deteriorated in their general condition and were sacrificed. The median survival was 53 days (Figure 6A). Whole-body necropsy displayed brittle bones, enlarged LNs, and splenomegaly, indicative of an aggressive hematopoietic disorder. Examination of PB from diseased Vav1 L-gp mice revealed a significant increase in WBC numbers (Figure 6B), with all subtypes in the PB, LNs, spleen, and BM characterized by ZsGreen expression, indicative of activation of gp130 signaling in the entire hematopoietic system (Supplemental Figure 7A). Accordingly, transcript analysis revealed L-gp130 expression and excessive STAT3 activation in splenocytes derived from Vav L-gp mice, while endogenous gp130 was nearly absent (Supplemental Figure 7B). However, no significant differences in the frequency of BM HSPCs were seen in diseased mice compared to age-matched controls by means of $\mathrm{Lin}^{-} \mathrm{Sca}^{+} \mathrm{c}-\mathrm{KIT}^{+}$and $\mathrm{Lin}^{-} \mathrm{S}$ $\mathrm{ca}^{-}{ }^{-} \mathrm{C}-\mathrm{KIT}^{+}$(multipotent progenitors) compartments (Supplemental Figure 7C). Flow cytometry analysis of LNs showed that the B cell compartment of these mice consisted of immature and pro-/pre-B cells (Figure 6 , $\mathrm{C}$ and $\mathrm{D}$ ) as well as mature $\mathrm{IgD}^{+} \mathrm{B}$ cells (Figure 6, C and E). Histological analysis revealed different B cell subpopulations in spleen and LNs of $\mathrm{Vav} 1 \mathrm{~L}$ - $g p$ mice: morphologically 1 resembled an aggressive B cell malignancy while the other represented a PC disorder (Figure 6F). Immunohistochemistry and flow cytometry showed scattered infiltration of T cells into LNs (Supplemental Figure 7, D and E). However, the main pathological feature was the massive accumulation of $\mathrm{CD} 138^{+} \mathrm{PCs}$ and $\mathrm{B} 220^{+} \mathrm{Pax} 5^{+} \mathrm{B}$ cells (Figure $6 \mathrm{~F}$ and Supplemental Figure 7E). The PC disorder in Vav1 L-gp mice was also supported by the presence of gammopathy in $100 \%$ of tested animals, however only to a moderate extent (Supplemental Figure 7, F and G). These findings are supported by results obtained from RNA-Seq analysis of Ig heavy chain constant regions (Supplemental Figure $7 \mathrm{H}$ ). Assessment of light chain constant region gene expression revealed significantly lower $\lambda$ gene profiles in Vav1 L-gp mice compared with WT controls (Supplemental Figure 7I).

These results are consistent with a dominant gp130 downstream signaling mechanism that enforces the development of B cell cancers even when activated at the HSPC stage.

Independent of the time point of activation during hematopoietic and B cell differentiation, all Cre $L$-gp compound mice succumbed to tumors of B cell origin. RNA-Seq analysis from tumors revealed clustering of Vav1 L-gp and CD19 L-gp mice (Supplemental Figure 8A). Comparison of CD19 L-gp tumors of the Mature and PC phenotypes displayed strong activation of WNT, BCR, and NF- $\mathrm{B}$ signaling and expression of gp130 downstream targets (Supplemental Figure 8B). Vav1 L-gp tumors, however, showed activation of all downstream cascades activated by IL-6/gp130 signaling (Supplemental Figure 8B).

\section{Discussion}

Tumor formation in all B cell Cre-transgenic models and a B cell tumor phenotype in Vav1Cre mice strongly supports the prominent role of gp130 signaling in mature B cell lymphomas, in particular MM (reviewed in ref. 41). Accordingly, and in support of the functional relevance and in vivo data, IL-6-transgenic mice on a C57BL/ 6 background exhibited plasmacytosis with infiltration into the spleen and LNs (19), whereas monoclonal and transplantable plasmacytomas developed only in IL-6 transgenic BALB/c mice (20). Several MM models are based on activation of the IL-6/IL-6R axis $(23,42,43)$, underscoring its importance in proliferation and malignant transformation of late B cells. These MM mouse models are limited because of their late disease onset or low penetrance. For example, expression of Myc in the GC using activation-induced deaminase led to MM development after 2 years (44), while IL-6-transgenic BALB/c mice succumbed to MM with a penetrance of only $40 \%$ at 12 months of age (45). Transgenic mice expressing 
A
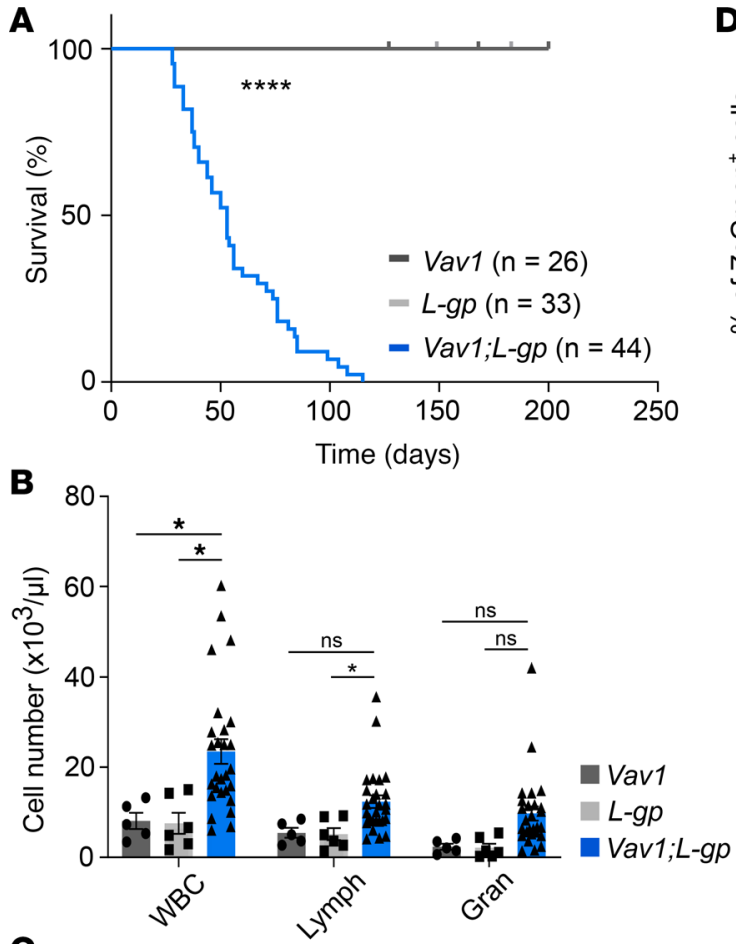

C

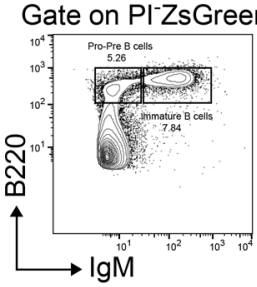

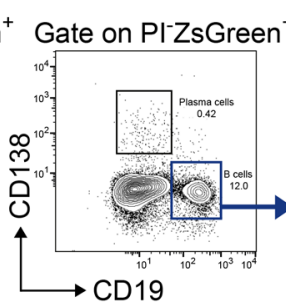

D

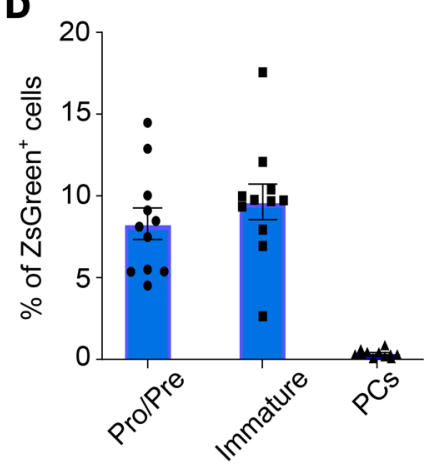

$\mathbf{E}$

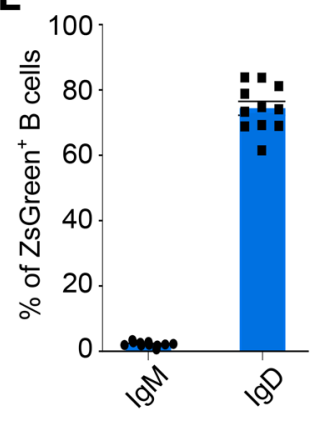

Gate on ZsGreen ${ }^{+}$BC
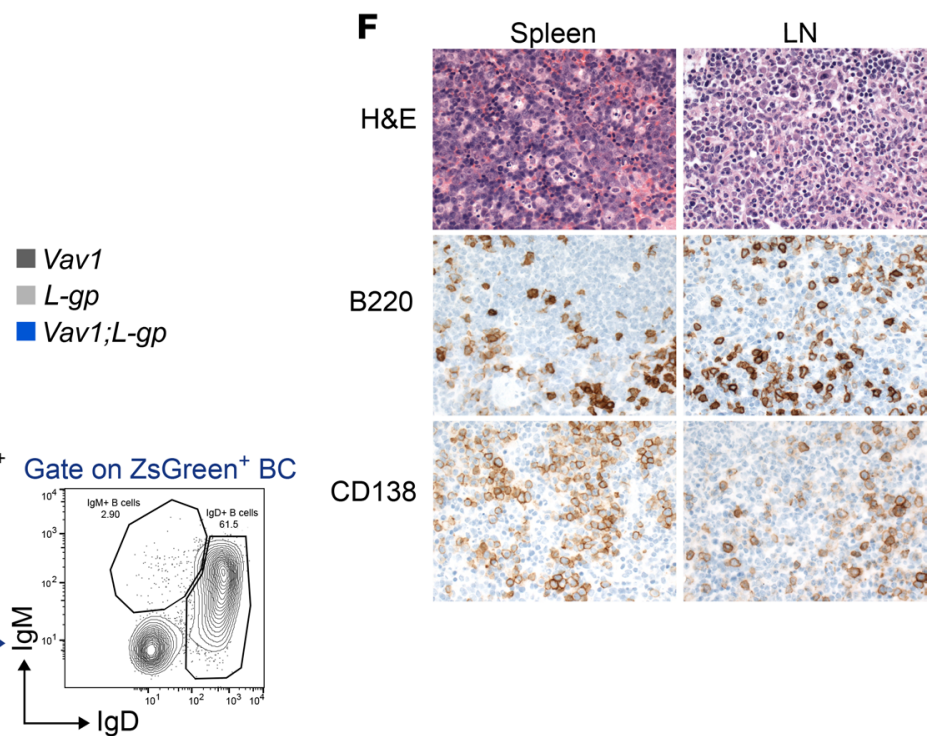

Figure 6. Conditional gp130 activation in hematopoietic stem/progenitor cells results in a highly aggressive B cell malignancy. (A) Kaplan-Meier curve showing survival of Vav1 L-gp mice $(n=44)$ in comparison with $\operatorname{Vav1}(n=26)$ and L-gp $(n=33)$ controls. Vav1 L-gp compound mice had a median survival of 53 days. Controls did not show any signs of malignancy within the observed time frame $\left({ }^{* * *} P<0.0001\right.$, Mantel-Cox test). (B) WBC analysis of PB in diseased Vav1 L-gp mice $(n=27)$ in comparison with normal Vav1 $(n=5)$ and L-gp $(n=6)$ controls $\left({ }^{*} P<0.05\right.$, 2-tailed Student's $t$ test). Shown are means \pm SEM. (C) Flow cytometric analysis of diseased Vav1 L-gp mice ( $n=11$ analyzed) for distinct B cell subsets. Cells were gated for PI-ZsGreen ${ }^{+}$living cells. Shown is the LN sample of 1 representative animal. Pro-/pre- and immature B cells (left) were present, while a small fraction of CD138+ PCs was seen (middle). The $\mathrm{CD}^{+} \mathrm{S}^{+} \mathrm{B}$ cell population was identified as a mature IgD+ differentiation state (right). (D) Quantitative analysis of LN infiltration by the distinct B cell subsets as shown in C from $n=11$ mice. Bars represent the mean percentage \pm SEM. The previous gate was set on ZsGreen+ living cells. (E) Quantitative analysis of $\mathrm{LN}$ infiltration by the distinct $B$ cell subsets as shown in C from $n=11$ mice. Bars represent the mean percentage \pm SEM. The previous gate was on ZsGreen ${ }^{+}$ living B cells. (F) Histological and immunohistochemical analysis from spleen (left) and LN (right) of a representative diseased Vav1 L-gp mouse. H\&E staining revealed high infiltration of tumor cells into both organs (upper row). The lower rows show B220+ and CD138+ expression; 11 mice were analyzed. Original magnification, $\times 400$.

high levels of XBP1, a transcription factor important for terminal B cell differentiation, mainly developed MGUS, and only few progressed into MM later in life (46).

In contrast with a previous study using $L$-gp 130 in a BM transduction-transplantation model in BAL$\mathrm{B} / \mathrm{c}$ mice, which are prone to developing PC disorders because of a mutation in the INK4a/ARF locus leading to partial disability of the tumor suppressor protein $\mathrm{p} 16^{\text {Ink4a }}(21)$, here we used a C57BL/6 genetic background. The development of a similar disease under these circumstances strongly supports a central role for gp130 signaling in mature B cell disorders, in particular MM. Altogether, the herein-presented conditional-knockin model for L-gp130 bears several advantages over published mouse models. First, it comes on the frequently used C57BL/ 6 background, allowing the generation of pure background compound transgenic mice in the context of targeted transgene activation, irrespective of mutations in the INK4a/ARF locus. Second, the model is targeted to B cells and eliminates the putative proinflammatory activation caused by STAT3 activation upon whole-BM infection. Third, L-gp130 BM and manifest tumors are transplantable, 
allowing additional gene editing of FL and BM cells in vitro with subsequent syngeneic transplantation. Fourth, beyond the context of tumor pathogenesis, it allows targeting of gp130/JAK/STAT3 activation to specific cellular components or tissues that are involved in inflammation and immunity, e.g., in arthritis (8).

At the time of diagnosis, $90 \%$ of MM patients present with monoclonal gammopathy protein in the serum or urine (47). In this study of a potentially novel gp130-driven mouse model, only one-third of mice with the PC phenotype but more than half of mice assigned to the Mature group showed this characteristic. Future studies are required to dissect these phenotypic differences in L-gp130-derived tumors. It has, however, been identified in various studies of human B cell lymphomas that paraproteins were produced not only in MM but also in subtypes of mature B cell lymphoma, including plasmablastic lymphoma, and in diffuse large B cell lymphoma (48-50).

IL-6/IL-6R/gp130 signaling is crucial for normal B cell differentiation and function, including the production of Ig by terminally differentiated PCs (11). In our study, enhanced signaling of this axis within the B cell lineage of young mice therefore resulted in an accumulation of mature $\mathrm{B}$ cell subsets, which then led to the activation of IL-6/JAK/STAT3, PI3K/AKT/mTOR, WNT, MYC, as well as BCR and NF-kB signaling pathways.

Immunization of mice expressing a dominant-negative form of gp130 led to a loss of antigen-specific antibody production without affecting GC development (51). This observation was partly supported by a study on immunized IL-6-deficient mice that presented with lower antigen-specific antibody titers but were additionally impaired in the formation of a normal GC. Thus, in the absence of IL-6, size and volume of GC B cells were severely diminished (17). These 2 studies point to the importance of IL-6/gp130 signaling for a functional GC reaction and subsequent Ig production by PCs. Here, we find that constitutive activation of gp130 signaling in the GC by means of $C \gamma 1 C$ re frequently resulted in an accumulation of CD $19^{+} \mathrm{GL} 7^{+} \mathrm{GC}$ B cells.

Although activation of gp130/JAK/STAT3 signaling occurs later in B cell differentiation in Blimp$1 C r e$ than in $C \gamma 1 C r e$ mice, a much shorter median survival was observed in the former, and tumor development was not restricted only to the PC phenotype. This might be explained by the fact that Blimp1 not only is expressed in B cells leaving the GC but also plays a role in the terminal differentiation of other cell subsets (52), and therefore gp130/JAK/STAT3 signaling in these mice is not restricted only to late B cells. However, even in Blimp1 L-gp mice, we saw the development of mature B cell diseases, including plasmacytoma, confirming the prominent role this pathway plays in B cells and their malignant transformation. Future studies on the Blimp1 L-gp cross will be needed that concentrate on the detailed characterization of this genotype.

In summary, we found that enforced B cell differentiation upon targeted gp130 signaling resulted in mature B cell lymphoma and plasmacytomas and was sufficient to completely abrogate activity of a B celltargeted Myc transgene. Gp130 signaling thus provides a selective growth and differentiation advantage for mature B cells and directs lymphomagenesis toward terminally differentiated B cell cancers.

\section{Methods}

Preparation and culture of MEFs. Preparation and culture of MEFs has been described elsewhere (53). Retroviral infection and analysis including primers used (Supplemental Table 1) is described in the Supplemental Methods.

Mice and transplantation experiments. All animal experiments were performed in accordance with Federation of European Laboratory Animal Science Associations (FELASA) guidelines and with the regional animal ethics committee approval (Regierung von Oberbayern, Munich, Germany). All strains used throughout this study were on a pure C57BL/6 background. The novel L-gp130 mouse line was generated by Mona Al-Maarri and Frank Thomas Wunderlich (Max Planck Institute for Metabolism Research, Center for Endocrinology, Preventive Medicine and Diabetes, Cologne, Germany), and Cre-transgenic strains were purchased from The Jackson Laboratory. Although for transplantation experiments only female mice were taken as recipients, both male and female mice were used for all the other investigations. Detailed information on mouse experiments is given in the Supplemental Methods.

Flow cytometry. Cells were stained in PBS (Thermo Fisher Scientific) containing 0.5\% BSA (Carl Roth $\mathrm{GmbH}$ ), and fluorescently labeled antibodies were used at 1:200 to 1:600 dilutions. Viability distinction was realized by staining with PI. Fluorescently labeled antibodies for extracellular staining are provided in the Supplemental Methods.

Histopathological analysis and immunohistochemistry. Tissue samples were fixed in $4 \%$ formaldehyde for 24-48 hours, paraffin embedded, sectioned, and stained using H\&E. Antibodies used for immunohistochemistry are listed in the Supplemental Methods. 
Isolation of genomic DNA from tumor material. Genomic DNA was isolated from tumor/tissue samples using the DNeasy Blood \& Tissue Kit according to the manufacturer's instructions (Qiagen), followed by analysis of IgH rearrangements as described in the Supplemental Methods.

$R N A$ isolation from tumor material for RNA-Seq. RNA was isolated from samples with an infiltration of greater than $60 \%$ ZsGreen ${ }^{+}$tumor cells using the RNeasy Plus Mini Kit (Qiagen) according to the manufacturer's protocols. Where infiltration rate was lower, $\mathrm{CD} 19^{+}$cells were collected by magnetic-activated cell sorting (MACS), and RNA was isolated thereafter followed by RNA-Seq and analysis of gene expression data as described in the Supplemental Methods.

Data deposition. The RNA-Seq data reported in this study are available from the National Center for Biotechnology Information's Gene Expression Omnibus with accession code GSE132148.

Statistics. All statistical tests were performed using the statistical functions of Excel (Microsoft) or GraphPad Prism 7. All values are expressed as mean \pm SEM, and a $P$ value less than 0.05 was considered significant $\left({ }^{*} P<0.05,{ }^{* *} P<0.005,{ }^{* *} P<0.0005\right.$, and $\left.{ }^{* * * *} P<0.0001\right)$. The 2-tailed Student's $t$ test was used to compare quantitative data between 2 independent samples. When comparing 3 or more groups, a 2-way ANOVA statistical test was used to analyze the differences between group means followed by Tukey's test. Survival analyses were performed using the Mantel-Cox test.

Study approval. All mice were bred and maintained in the animal facility of Technische Universität München under specific pathogen-free conditions. Animal experiments were performed in accordance with the FELASA guidelines and with approval from the regional animal ethics committee (Regierung von Oberbayern, Munich, Germany)

\section{Author contributions}

MAM generated the transgenic L-gp130 mouse strain while AKS conducted the majority of experiments shown in this study and therefore shares the first authorship. RÖ, MS, MT, and KP performed and interpreted experiments. LQM, IGM, MM, and KS analyzed and interpreted histological and immunohistochemical data. HCM analyzed and interpreted the gene expression data. AKS, MAM, SM, RR, FTW, SRJ, and UK designed the study and with JR and SS interpreted the data. AKS, MAM, FTW, SRJ, and UK wrote the manuscript. All authors critically reviewed and approved the final manuscript.

\section{Acknowledgments}

We thank Katharina Collins, Kerstin Behnke, Jolanta Slawska, Olga Seelbach, Markus Utzt, Philipp Jost, Maike Kober, Valeria Soberón, Tim Ammon, and Marc Schmidt-Supprian (Technische Universität München, Munich, Germany) for their expert opinions and technical support. We acknowledge Life Science Editors for editorial advice. UK received support from Deutsche Krebshilfe (grants 111305 and 111944) and Deutsche Forschungsgemeinschaft (SFB 824 and SFB 1335). UK was further supported by Stiftung Charité. SRJ was supported by the Deutsche Forschungsgemeinschaft (SFB 841).

Address correspondence to: Ulrich Keller, Department of Hematology, Oncology and Tumor Immunology (Campus Benjamin Franklin), Charité - Universitätsmedizin Berlin, Hindenburgdamm 30, 12203 Berlin, Germany. Phone: 49.30.450.513381; Email: ulrich.keller@charite.de.

1. Hibi M, Murakami M, Saito M, Hirano T, Taga T, Kishimoto T. Molecular cloning and expression of an IL-6 signal transducer, gp130. Cell. 1990;63(6):1149-1157.

2. Nish SA, et al. T cell-intrinsic role of IL-6 signaling in primary and memory responses. Elife. 2014;3:e1949.

3. Yu H, Pardoll D, Jove R. STATs in cancer inflammation and immunity: a leading role for STAT3. Nat Rev Cancer. 2009;9(11):798-809.

4. Jones SA, Scheller J, Rose-John S. Therapeutic strategies for the clinical blockade of IL-6/gp130 signaling. J Clin Invest. 2011;121(9):3375-3383.

5. Scheller J, Chalaris A, Schmidt-Arras D, Rose-John S. The pro- and anti-inflammatory properties of the cytokine interleukin-6. Biochim Biophys Acta. 2011;1813(5):878-888.

6. Schaper F, Rose-John S. Interleukin-6: biology, signaling and strategies of blockade. Cytokine Growth Factor Rev. 2015;26(5):475-487.

7. Silver JS, Hunter CA. gp130 at the nexus of inflammation, autoimmunity, and cancer. J Leukoc Biol. 2010;88(6):1145-1156.

8. Garbers C, Heink S, Korn T, Rose-John S. Interleukin-6: designing specific therapeutics for a complex cytokine. Nat Rev Drug Discov. 2018;17(6):395-412.

9. Benekli M, Baer MR, Baumann H, Wetzler M. Signal transducer and activator of transcription proteins in leukemias. Blood. 2003;101(8):2940-2954. 
10. Yoshizaki K, Nakagawa T, Fukunaga K, Tseng LT, Yamamura Y, Kishimoto T. Isolation and characterization of B cell differentiation factor (BCDF) secreted from a human B lymphoblastoid cell line. J Immunol. 1984;132(6):2948-2954.

11. Hirano T, et al. Complementary DNA for a novel human interleukin (BSF-2) that induces B lymphocytes to produce immunoglobulin. Nature. 1986;324(6092):73-76.

12. Nishimoto $\mathrm{N}$, et al. Oncostatin M, leukemia inhibitory factor, and interleukin 6 induce the proliferation of human plasmacytoma cells via the common signal transducer, gp130. J Exp Med. 1994;179(4):1343-1347.

13. Larousserie F, Charlot P, Bardel E, Froger J, Kastelein RA, Devergne O. Differential effects of IL-27 on human B cell subsets. J Immunol. 2006;176(10):5890-5897.

14. Silver JS, Hunter CA. gp130 at the nexus of inflammation, autoimmunity, and cancer. J Leukoc Biol. 2010;88(6):1145-1156.

15. Kopf M, et al. Impaired immune and acute-phase responses in interleukin-6-deficient mice. Nature. 1994;368(6469):339-342.

16. Kumanogoh A, et al. Impairment of antigen-specific antibody production in transgenic mice expressing a dominant-negative form of gp130. Proc Natl Acad Sci USA. 1997;94(6):2478-2482.

17. Kopf M, Herren S, Wiles MV, Pepys MB, Kosco-Vilbois MH. Interleukin 6 influences germinal center development and antibody production via a contribution of C3 complement component. J Exp Med. 1998;188(10):1895-1906.

18. Hunter CA, Jones SA. IL-6 as a keystone cytokine in health and disease. Nat Immunol. 2015;16(5):448-457.

19. Suematsu S, et al. IgG1 plasmacytosis in interleukin 6 transgenic mice. Proc Natl Acad Sci USA. 1989;86(19):7547-7551.

20. Suematsu S, et al. Generation of plasmacytomas with the chromosomal translocation $\mathrm{t}(12 ; 15)$ in interleukin 6 transgenic mice. Proc Natl Acad Sci USA. 1992;89(1):232-235.

21. Zhang SL, et al. Efficiency alleles of the Pctr1 modifier locus for plasmacytoma susceptibility. Mol Cell Biol. 2001;21(1):310-318.

22. Stuhlmann-Laeisz C, et al. Forced dimerization of gp130 leads to constitutive STAT3 activation, cytokine-independent growth, and blockade of differentiation of embryonic stem cells. Mol Biol Cell. 2006;17(7):2986-2995.

23. Dechow T, et al. GP130 activation induces myeloma and collaborates with MYC. J Clin Invest. 2014;124(12):5263-5274.

24. Hallek M, Bergsagel PL, Anderson KC. Multiple myeloma: increasing evidence for a multistep transformation process. Blood. 1998;91(1):3-21.

25. Huang X, et al. Activation of the STAT3 signaling pathway is associated with poor survival in diffuse large B-cell lymphoma treated with R-CHOP. J Clin Oncol. 2013;31(36):4520-4528.

26. Crescenzo R, et al. Convergent mutations and kinase fusions lead to oncogenic STAT3 activation in anaplastic large cell lymphoma. Cancer Cell. 2015;27(4):516-532.

27. Tiacci E, et al. Pervasive mutations of JAK-STAT pathway genes in classical Hodgkin lymphoma. Blood. 2018;131(22):2454-2465.

28. Guang MHZ, et al. Overcoming multiple myeloma drug resistance in the era of cancer 'omics'. Leuk Lymphoma. 2018;59(3):542-561.

29. Rickert RC, Roes J, Rajewsky K. B lymphocyte-specific, Cre-mediated mutagenesis in mice. Nucleic Acids Res. 1997;25(6):1317-1318.

30. Barberis A, Widenhorn K, Vitelli L, Busslinger M. A novel B-cell lineage-specific transcription factor present at early but not late stages of differentiation. Genes Dev. 1990;4(5):849-859.

31. Casola S, et al. Tracking germinal center B cells expressing germ-line immunoglobulin gamma1 transcripts by conditional gene targeting. Proc Natl Acad Sci USA. 2006;103(19):7396-7401.

32. Kallies A, et al. Initiation of plasma-cell differentiation is independent of the transcription factor Blimp-1. Immunity. 2007;26(5):555-566.

33. Dominguez-Sola D, et al. The proto-oncogene MYC is required for selection in the germinal center and cyclic reentry. Nat Immunol. 2012;13(11):1083-1091.

34. Reddy A, et al. Genetic and functional drivers of diffuse large B cell lymphoma. Cell. 2017;171(2):481-494.e15.

35. Chapuy B, et al. Molecular subtypes of diffuse large B cell lymphoma are associated with distinct pathogenic mechanisms and outcomes. Nat Med. 2018;24(5):679-690.

36. Adams JM, et al. The c-myc oncogene driven by immunoglobulin enhancers induces lymphoid malignancy in transgenic mice Nature. 1985;318(6046):533-538.

37. Munoz J, Dhillon N, Janku F, Watowich SS, Hong DS. STAT3 inhibitors: finding a home in lymphoma and leukemia. Oncologist. 2014;19(5):536-544.

38. Sansone P, Bromberg J. Targeting the interleukin-6/Jak/stat pathway in human malignancies. J Clin Oncol. 2012;30(9):1005-1014

39. Ogilvy S, Elefanty AG, Visvader J, Bath ML, Harris AW, Adams JM. Transcriptional regulation of vav, a gene expressed throughout the hematopoietic compartment. Blood. 1998;91(2):419-430.

40. Ogilvy S, Metcalf D, Gibson L, Bath ML, Harris AW, Adams JM. Promoter elements of vav drive transgene expression in vivo throughout the hematopoietic compartment. Blood. 1999;94(6):1855-1863.

41. Burger R. Impact of interleukin-6 in hematological malignancies. Transfus Med Hemother. 2013;40(5):336-343.

42. Rossi M, Botta C, Arbitrio M, Grembiale RD, Tagliaferri P, Tassone P. Mouse models of multiple myeloma: technologic platforms and perspectives. Oncotarget. 2018;9(28):20119-20133.

43. Paton-Hough J, Chantry AD, Lawson MA. A review of current murine models of multiple myeloma used to assess the efficacy of therapeutic agents on tumour growth and bone disease. Bone. 2015;77:57-68.

44. Chesi M, Bergsagel PL. Many multiple myelomas: making more of the molecular mayhem. Hematology Am Soc Hematol Educ Program. 2011;2011:344-353.

45. Kovalchuk AL, et al. IL-6 transgenic mouse model for extraosseous plasmacytoma. Proc Natl Acad Sci USA. 2002;99(3):1509-1514.

46. Carrasco DR, et al. The differentiation and stress response factor XBP-1 drives multiple myeloma pathogenesis. Cancer Cell. 2007;11(4):349-360.

47. International Myeloma Working Group. Criteria for the classification of monoclonal gammopathies, multiple myeloma and related disorders: a report of the International Myeloma Working Group. Br J Haematol. 2003;121(5):749-757.

48. Maniatis A. Pathophysiology of paraprotein production. Ren Fail. 1998;20(6):821-828.

49. Dang M, Gajendra S, Goel S, Jha B, Sahni T, Sachdev R. Diffuse large B cell lymphoma with high M protein: an unusual finding. Blood Res. 2015;50(1):61-62.

50. Rosean TR, et al. KSHV-encoded vIL-6 collaborates with deregulated c-Myc to drive plasmablastic neoplasms in mice. Blood 
Cancer J. 2016;6:e398.

51. Kumanogoh A, et al. Impairment of antigen-specific antibody production in transgenic mice expressing a dominant-negative form of gp130. Proc Natl Acad Sci USA. 1997;94(6):2478-2482.

52. Xin A, Nutt SL, Belz GT, Kallies A. Blimp1: driving terminal differentiation to a T. Adv Exp Med Biol. 2011;780:85-100.

53. Jozefczuk J, Drews K, Adjaye J. Preparation of mouse embryonic fibroblast cells suitable for culturing human embryonic and induced pluripotent stem cells. J Vis Exp. 2012;(64):3854. 